Meta

Journal des tradlucteurs

Translators' Journal

\title{
Calques et créations linguistiques
}

\section{Michel Chansou}

Volume 29, numéro 3, septembre 1984

URI : https://id.erudit.org/iderudit/002216ar

DOI : https://doi.org/10.7202/002216ar

Aller au sommaire du numéro

Éditeur(s)

Les Presses de l'Université de Montréal

ISSN

0026-0452 (imprimé)

1492-1421 (numérique)

Découvrir la revue

Citer cet article

Chansou, M. (1984). Calques et créations linguistiques. Meta, 29(3), 281-284.

https://doi.org/10.7202/002216ar d'utilisation que vous pouvez consulter en ligne.

https://apropos.erudit.org/fr/usagers/politique-dutilisation/ 


\section{ÉTUDES \\ TERMINOLOGIQUES \\ ET LINGUISTIQUES}

\section{CALQUES ET CRÉATIONS LINGUISTIQUES}

Les langues scientifiques et techniques en français contemporain doivent faire face à des besoins massifs de dénominations nouvelles. Compte tenu de la position dominante de la langue anglo-américaine et des exigences de la communication internationale, convient-il de privilégier d'une façon systématique le procédé du calque pour désigner des réalités techniques empruntées. N'est-il pas préférable dans certains cas d'avoir recours à des créations linguistiques indigènes réalisées à partir des ressources propres de la langue ? Calques ou créations linguistiques indigènes? Le choix entre ces deux procédés auquel sont confrontés quotidiennement les traducteurs techniques et les terminologues est lié à des prises de position théoriques et à des options linguistiques, mais dépend aussi, dans la pratique, de la diversité des conditions sociales de la communication. Les quelques réflexions que nous présentons ici à ce sujet sont tirées d'une recherche que nous avons consacrée aux travaux d'un organisme de terminologie, le Comité d'étude des termes techniques français (CETTF) ${ }^{1}$.

\section{QUELQUES DÉFINITIONS}

Nous retiendrons tout d'abord la définition du calque donnée par Einar Haugen dans le cadre de sa description des mécanismes de l'emprunt linguistique ${ }^{2}$. Haugen distingue trois types d'emprunt selon le degré de substitution de la forme étrangère dans la langue emprunteuse :

- L'emprunt intégral (loan word) . La forme étrangère est entièrement adoptée par la langue emprunteuse ; il n'y a donc aucune substitution de forme.

- L'emprunt partiel ou emprunt hybride (loan blend), dans lequel se réalise une substitution partielle : un élément de la forme étrangère est adopté, l'autre élément est remplacé par un élément de la langue emprunteuse.

- L'emprunt remanié (loan-shift) . La forme étrangère est entièrement remplacée par une forme de la langue emprunteuse ; la substitution est totale.

1. Recherche sur la planification linguistique en français contemporain. Le Comité d'étude des termes techniques français et l'emprunt linguistique, thèse de $3^{\mathrm{e}}$ cycle, Université Paris-Nord, 1981 (document multigraphié).

2. "The analysis of linguistic borrowing ", dans Langages, 26, 1950, pp. 210-231. 
Le calque (loan translation), qui appartient à la classe des emprunts remaniés, représente pour Haugen une extension du processus observé dans la réalisation de l'emprunt

Instead of substituting only one half of the word, the borrowers have here analyzed and substituted both halves. They have imported a particular structural pattern, viz. the combination of the two constituents into a compound expression with a new meaning of its own not derivable by a simple addition of the two parts (p. 214).

Le mécanisme du calque repose donc sur une double opération : a) l'analyse des éléments d'une forme étrangère (forme d'une unité lexicale complexe ou d'une unité construite par dérivation), b) le remplacement des deux éléments de cette forme par deux éléments correspondants de la langue emprunteuse. Le calque est la reproduction exacte d'un modèle étranger, une traduction littérale. Ainsi le CETTF propose pour trapped water, terme du vocabulaire de l'exploitation des forages, l'équivalent eau piégée, pour dog leg (vocabulaire de l'industrie du pétrole) l'équivalent patte de chien, pour brushability (vocabulaire de la technique des peintures) l'équivalent brossabilitée $e^{3}$. Le calque apparaît comme une forme atténuée de l'emprunt, facilement assimilable dans la langue emprunteuse, et il facilite la communication internationale dans les domaines scientifiques et techniques, dans la mesure où des structures lexicales identiques introduites dans plusieurs langues peuvent être aisément reconnues par des spécialistes.

La définition d'Haugen, toutefois, en raison même de sa précision, ne permet pas de rendre compte de tous les degrés du calque. II n'est pas toujours possible de reproduire exactement les éléments d'une forme étrangère pour réaliser ce que nous pouvons appeler un calque littéral. Pour que la forme construite par imitation puisse plus facilement être adoptée par la langue emprunteuse, on est amené à modifier un des éléments de la forme étrangère, ou on a recours à un énoncé traductif plus ou moins fidèle qui cherche à rendre d'une façon plus élégante ou plus précise aussi bien le modèle de la forme que le contenu du concept. Nous citerons, par exemple, homme de terrain pour field worker, liste d'adresses pour mailing list, premier crayon pour pencil test, moyenne de temps de bon fonctionnement pour mean time between failures. On s'éloigne ainsi progressivement du calque littéral, et nous désignerons tous ces énoncés traductifs, qui représentent divers degrés de liberté par rapport au modèle, par l'expression calque aménagé. Ces deux types de calques constituent l'ensemble des calques de forme ${ }^{4}$. Toutes les constructions de formes nouvelles appartenant à cet ensemble ont pour caractère commun le fait qu'elles reproduisent, soit exactement, soit approximativement, le modèle d'une forme étrangère.

En poursuivant notre analyse, nous sommes amené à considérer un type de formes construites sensiblement différent du calque de forme tel que nous venons de le définir. Il arrive que des formes soient créées dans la langue pour désigner un concept emprunté, sans que s'exerce l'influence de la forme étrangère déjà associée à ce concept. Ces créations appartiennent bien évidemment à la classe des emprunts " remaniés " (au sens de Haugen), puisqu'elles constituent le signifiant d'un nouveau signe dont le signifié est emprunté. Mais elles ne peuvent pas être considérées, à proprement parler, comme des calques, puisqu'elles ne reproduisent pas la forme du modèle étranger. Le CETTF, par exemple, pour traduire le terme coke car (wagon recueillant le coke extrait des fours de carbonisation pour le conduire sous la tour d'extinction) propose deux

3. La plupart des exemples cités dans cet article figurent dans l'ensemble terminologique étudié par le CETTF. Voir Termes techniques français (1972) : Essai d'orientation de la technologie, établi par le Comité d'étude des termes techniques français, Paris, Hermann.

4. Nous n'aborderons pas ici l'étude des calques de sens, ou calques sémantiques. 
équivalents : chariot à coke et wagon d'extinction. Si le premier de ces équivalents est un calque littéral, on voit que le second, wagon d'extinction, ne reproduit en aucune façon le modèle de la forme anglaise ; il représente une forme entièrement nouvelle constituant une définition abrégée du concept. De même, pour wildcat , terme du vocabulaire du pétrole désignant un sondage effectué dans une zone incomplètement explorée, le CETTF propose, à la place du calque chat sauvage, l'équivalent sondage d'exploration.

\section{LES CONDITIONS D'EMPLOI DES CALQUES ET DES CRÉATIONS LINGUISTIQUES}

Pour analyser les conditions dans lesquelles s'opèrent les choix entre calques et créations linguistiques, nous examinerons tout d'abord les principes linguistiques qui déterminent l'orientation des travaux du CETTF. Les vocabulaires techniques doivent satisfaire à une double exigence : $a$ ) les termes utilisés doivent désigner d'une façon précise les objets et les concepts techniques, ils doivent être clairs de façon que les langues techniques soient, en principe, accessibles à tous ; $b$ ) le développement actuel des sciences et des techniques rend nécessaire l'internationalisation des terminologies. Ces deux exigences sont parfois incompatibles, et le CETTF n'arrive pas toujours à les concilier. Il cherche à maintenir, chaque fois que cela se peut, le caractère international d'un terme, et il utilise largement le procédé du calque qui rend plus facile la communication entre techniciens de différents pays. Mais, dans certains cas, il estime qu'il n'est pas possible d'introduire dans la langue, par la reproduction d'un modèle étranger, des dénominations obscures ou imprécises, et il crée alors à partir des ressources propres de la langue une forme nouvelle.

Ainsi, pour traduire le terme chemurgy désignant un ensemble de techniques utilisant comme matières de base des produits agricoles, il écarte le calque chimiurgie parce qu'il est, comme le terme américain, un terme impropre. Il résulte en effet de la définition de chemurgy que ce terme ne désigne pas nécessairement une technique chimique. Chimiurgie « oriente à tort l'esprit vers la chimie et reste muet sur l'élément agricole, qui est commun à toutes les techniques rassemblées sous le vocable chemurgy ". Le CETTF construit donc le néologisme agrotechnie. De la même façon, il remet en cause plusieurs dénominations qui sont mal adaptées au concept qu'elles désignent. À propos de photo timer (cellule photoélectrique agissant directement sur le diaphragme d'un objectif photographique), il constate que le terme timer est ici employé sans raison, et il propose comme équivalent posemètre automatique, dénomination entièrement construite à partir de l'analyse du concept. Pour traduire silent block, il cherche un terme qui puisse désigner d'une façon satisfaisante ce type d'articulation élastique " dont la caractéristique essentielle est moins le silence que l'amortissement des vibrations ", et il retiendra support élastique ou articulation élastique. Nous voyons ici que les créations linguistiques indigènes représentent un procédé linguistique qui s'oppose d'une façon significative aux calques de forme.

D'autre part, lorsque le calque d'une expression étrangère risque de n'être compris que par un petit nombre de techniciens, le CETTF préfère créer une dénomination nouvelle qui rendra compte clairement du concept. Pour traduire, par exemple, localizer (vocabulaire de l'aéronautique), pack shot (vocabulaire de la publicité et de la télévision) ${ }^{5}$, wild cat (vocabulaire de l'industrie du pétrole), il propose à la place de calques les dénominations radioalignement de piste, plan final, sondage d'exploration. On remarquera que ces innovations lexicales que nous avons appelées pour les distinguer du calque des traductions " libres", puisqu'elles ne reproduisent en aucune façon la forme du modèle étranger, sont en réalité soumises à des exigences beaucoup plus contraignantes,

5. Gros plan sur le produit ou sur la marque à la fin d'un film publicitaire. 
dans la mesure où elles doivent être adaptées d'une façon aussi précise que possible au concept qu'elles désignent. De telles créations linguistiques ne représentent pas ici un choix neutre ; elles apparaissent comme l'application de principes linguistiques et constituent un des éléments de l'action menée par le CETTF en vue d'aménager les langues techniques.

Aurélien Sauvageot, qui s'est beaucoup intéressé aux travaux du CETTF, a abordé à plusieurs reprises le problème posé par l'usage des calques et des créations d'origine française $^{6}$. Il estime, pour sa part, qu'il faut éviter de construire des dénominations "françaises " pour désigner des concepts techniques qui ont déjà reçu une appellation dans une autre langue, et qu'on doit avoir recours au calque le plus souvent possible. Le terme d'origine et le calque ont, en effet, pour le spécialiste la même motivation. Si l'on substitue au calque un terme français issu d'une motivation différente, on risque de ne pas être compris par les techniciens. Ainsi le recours à une terminologie proprement française constituerait sans doute un écran presque infranchissable pour tous ceux qui ont été initiés à la terminologie d'origine. Dans le contexte scientifique et technique international actuel, l'utilisation du calque relève de la nécessité pratique de normaliser les terminologies, et l'on ne peut pas se permettre le luxe de développer des terminologies à caractère national, même si elles répondent mieux dans certains cas à des exigences de clarté et de précision.

Les positions de Sauvageot sont ici, on le voit, sensiblement différentes de celles du CETTF. Mais on remarquera que ces deux conceptions ne font pas référence, en réalité, à une même situation de communication technique. Les arguments de Sauvageot s'appliquent principalement à une communication spécialisée entre des techniciens appartenant à un même domaine d'activité et se situant à un certain niveau de compétences techniques. (Il ne s'agit pas de la communication établie à l'intérieur d'un atelier entre simples techniciens.) Les travaux du CETTF se rapportent à un niveau de communication technique plus général et concernent en particulier les différents degrés de vulgarisation scientifique et technique et les usages banalisés des vocabulaires techniques dans la langue courante.

On peut observer d'autre part que Sauvageot comme les membres du CETTF insiste sur la nécessité de proposer aux locuteurs des termes bien motivés, de façon à assurer la transparence de la communication. Or il apparaît que la valeur de la motivation d'un terme technique peut dépendre du niveau d'information des locuteurs et varier en fonction des conditions sociales de telle ou telle situation de communication. Prenons l'exemple du terme wildcat que nous avons déjà cité. Pour des techniciens de l'industrie du forage pétrolier le calque chat sauvage est parfaitement motivé par rapport à la forme du modèle américain. La relation métaphorique à partir de laquelle cette dénomination est construite (le wildcat est un appareil de forage installé à l'écart, semblable à un chat sauvage chassant loin de ses congénères) est aisément perçue par ces techniciens, puisqu'ils connaissent déjà les caractéristiques de la technique ainsi désignée. La dénomination chat sauvage est donc tout à fait transparente, mais elle ne l'est vraiment que dans une communication établie entre techniciens d'un même domaine. Dans une autre situation, pour un locuteur qui n'est pas informé des techniques du forage pétrolier, qu'il soit spécialiste d'un autre domaine ou non technicien, chat sauvage employé sans référence au modèle américain et en dehors d'un contexte explicatif précis, est un terme non motivé qui introduit un élément d'opacité dans la communication. À l'inverse,

6. Cf. "La langue française et les nécessités de l'expression scientifique et technique ", dans Vie et Langage, $\mathrm{n}^{\circ} 175$, octobre 1966, et $\mathrm{n}^{\circ} 176$, novembre 1966. Voir également Portrait du vocabulaire français, Paris, Larousse, 1964, pp. 254, 255 et 264. 
l'équivalent de construction française sondage d'exploration, qui est relativement clair et motivé pour un locuteur non spécialiste, risque d'être pour les spécialistes du domaine considéré une expression obscure ou sans signification précise, dans la mesure où ceux-ci n'auront pas été préalablement informés de l'équivalence établie entre le terme wildcat et cette nouvelle dénomination. On peut penser que de toute façon les dénominations wildcat et chat sauvage seront toujours pour eux mieux motivées et d'un emploi plus naturel parce qu'elles sont mieux adaptées à la réalité sociale et aux exigences d'une certaine situation de communication.

On pourrait faire des remarques semblables à propos des traductions de pack shot (plan-paquet ou plan final), de tracer (traceur ou indicateur radio-actif), de tracking (dépistage ou localisation dynamique), etc. Les notions de motivation et de transparence sont des notions relatives, et un même équivalent peut être motivé ou non motivé, transparent ou obscur, selon les situations. Ainsi les conceptions du CETTF et de Sauvageot nous semblent devoir être nuancées en fonction d'une approche plus proprement sociolinguistique du problème posé.

\section{CONCLUSION}

L'utilisation des calques et des créations linguistiques indigènes dans les langues techniques, comme l'utilisation de tout autre procédé de création lexicale, est donc liée aux conditions de la communication technique et aux contraintes propres à chaque situation. Leur implantation dans l'usage repose non seulement sur des mécanismes linguistiques, mais aussi sur des processus d'ordre social.

Les terminologies presque entièrement construites à partir du calque ou à partir de la création indigène présentent souvent un caractère artificiel. On peut se demander s'il est souhaitable d'employer systématiquement l'un ou l'autre de ces procédés dans les travaux de traduction et de normalisation, bien qu'il soit souvent difficile d'éviter cette systématisation, en particulier pour les vocabulaires très spécialisés. Quoi qu'il en soit, il apparaît que le développement d'une terminologie dépend de multiples facteurs économiques, psycholinguistiques et sociaux, et que les exigences de la communication internationale comme le souci de préserver la clarté de la langue ne sont pas les seuls principes à retenir pour orienter les travaux terminologiques. Dans la perspective d'un aménagement " raisonné " des langues techniques, les terminologues et les normalisateurs devraient pouvoir s'appuyer sur des descriptions méthodiques des divers usages sociaux des vocabulaires techniques et sur une analyse des facteurs qui favorisent l'implantation des néologismes dans la langue. De nombreuses recherches restent à entreprendre pour rendre compte de ces différents aspects. 